\title{
Uso de fototerapia en niños: nuestra experiencia
}

\author{
Delia-Stella Ibáñez-Gómez D MD'; Hernán Moumdjian MD²
}

\section{RESUMEN}

Introducción: la fototerapia utilizada en adultos hace más de 30 años ${ }^{(1)}$ es eficaz y segura. También es útil en dermatosis pediátricas como alternativa al tratamiento tópico o sistémico.

Objetivo: compartir nuestra experiencia con el uso de fototerapia en niños.

Resultados: la radiación UVB NB fue más utilizada que la UVA1 y la PUVA.

Conclusiones: el vitíligo, la psoriasis y la dermatitis atópica fueron las dermatosis más frecuentemente tratadas.

PALABRAS CLAVE: niños; radiación ultravioleta B; PUVA; fototerapia.

\section{USE OF PHOTOTHERAPY IN CHILDREN: OUR EXPERIENCE}

\section{SUMMARY}

Introduction: Phototherapy used in adults more than 30 years ago ${ }^{(1)}$ is effective and safe. Useful in pediatric dermatoses as an alternative to topical or systemic treatment.

Objective: Share our experience with the use of phototherapy in children.

Results: UVB NB radiation was more used than UVA1 and PUVA.

Conclusions: Vitiligo, psoriasis and atopic dermatitis were the most frequently treated dermatoses.

KEY WORDS: children; ultraviolet B radiation; PUVA; phototherapy.

1. Médica asociada clínica pediátrica Hospital Italiano de Buenos Aires, Argentina. Dermatóloga Pediatra Centro Galeno Barrio Norte, Buenos Aires, Argentina. ORCID https://orcid.org/oooo-0001-8305-7595

2. Médico Dermatólogo, Obra Social del Personal de Dirección de Sanidad Luis Pasteur Buenos Aires, Argentina. ORCID https://orcid.org/oooo-0003-0083-2133

Correspondencia: Delia Stella Ibáñez Gómez; email: ibanezmoumdjianspeedy@gmail.com

Recibido: 10/05/19; aceptado: 13/08/19

Cómo citar: Ibáñez-Gómez DS, Moumdjian H. Uso de fototerapia en niños: nuestra experiencia. Rev Asoc Colomb Dermatol. 2020;28(1):63-74. DOI: https://doi.org/10.29176/2590843X.1491

Financiación: ninguna, conflictos de interés: ninguno 


\section{INTRODUCCIÓN}

La fototerapia es una herramienta eficaz utilizada en adultos desde hace más de 30 años para tratamiento local de enfermedades dermatológicas como psoriasis, liquen plano y micosis fungoide 1. Posee un buen perfil de seguridad, lo que resulta de gran utilidad en niños, ya que permite el manejo de patologías que no han respondido a tratamientos tópicos o como una opción previa a un tratamiento sistémico ${ }^{(2)}$. Está basada en el uso de fotones que actúan en la piel en diferentes niveles de profundidad dependiendo de su longitud de onda. Existen distintos tipos de radiación ultravioleta (RUV): UVA (320-400 nm), UVA de banda angosta (UVA1; $410 \mathrm{~nm}$ ), UVB de banda ancha o broad band (UVB BB; 280-320 nm) y UVB banda angosta o narrow band (UVB NB; $311 \mathrm{~nm}$ ). La UVB llega a la epidermis, mientras que la UVA alcanza la dermis profunda. La PUVA, la utilización de UVA más psoraleno, aumenta su efecto fotosensibilizador (3). Otras terapias, como el láser de excimer (308 nm), son útiles en áreas pequeñas, vitíligo ${ }^{(4)}$, psoriasis en placa leve a moderada en adultos, alopecia areata (AA), como reportan Ohtsuki y colaboradores ${ }^{(5)}$, y requiere que el paciente permanezca inmóvil y colabore. Abrouk y colaboradores 6 indican que es un procedimiento bien aceptado, con pocos efectos adversos en niños, como la hiperpigmentación.

La fototerapia posee un efecto antiinflamatorio, inmunomodulador, antiproliferativo y apoptótico que modifica el eritema, la descamación, la induración y el prurito en la psoriasis y dermatitis atópica. Su rol estimulador de la melanogénesis permite la pigmentación de las máculas acrómicas en el vitíligo ${ }^{(7)}$.

Reportes del uso de fototerapia en adultos ${ }^{(8-19)}$ brindan información útil para la implementación en niños, ya que pocos trabajos miden la eficacia y la seguridad en esta población (20-26). En Argentina, se destacan Ubogui y colaboradores ${ }^{(27)}$ y Cervini y colaboradores (28), quienes describieron una serie de 14 niños con micosis fungoides tratados con fototerapia; de estos, 8 recibieron UVB, otros PUVA o UVA1. Ibáñez y Moumdjian ${ }^{(29)}$ comparten su experiencia de 10 años de uso con este tratamiento. A nivel mundial, Tan y colaboradores ${ }^{(30)}$ describieron su experiencia con el uso de UVB NB en un trabajo prospectivo de 15 años que incluyó 116 niños, donde encontraron que la fototerapia fue efectiva y bien tolerada. Percivalle y colaboradores (31) realizaron una evaluación en 28 pacientes con vitíligo y encontraron una respuesta excelente en el 14\% y buena en el 28,6\% de estos, con buena adherencia. Koh y colaboradores ${ }^{(26)}$ estudiaron retrospectivamente a un grupo de 71 pacientes con vitíligo, en quienes la fototerapia mostró una buena respuesta.

La fototerapia está indicada en dermatosis con compromiso superficial o profundo, como: Psoriasis, vitíligo, dermatitis atópica, eczemas, prurigo (simple, crónico, solar), esclerodermia localizada y sistémica, pitiriasis liquenoide et varioliforme acuta (PLEVA), pitiriasis liquenoide crónica (PLC), enfermedad del injerto versus huésped (EIVH), alopecia areata, liquen escleroso (LE), plano y lineal, micosis fungoide (MF) estadios IA-IB, erupción polimorfa lumínica, papulosis linfomatoide, granuloma anular diseminado, urticaria crónica, mastocitosis cutánea maculopapular, fotodermatosis, pitiriasis rubra pilaris, pitiriasis rosada extendida, entre otros. En la tabla 1 se especifica el mecanismo de acción de acuerdo con cada patología ${ }^{(7)}$.

Los efectos adversos pueden ser agudos (quemaduras, eritema y prurito) o crónicos (fotodaño y carcinogénesis). De ahí la importancia del buen uso y la fotoeducación ${ }^{(32,33)}$.

Los pacientes pediátricos no son "adultos en miniatura” y por su condición y la cronicidad de su patología se debe elegir cuidadosamente el tratamiento que será administrado. La fototerapia es un recurso con demanda y utilidad en crecimiento. Se conoce su efectividad, pocos efectos adversos, fácil implementación, buena adherencia y permiten su reutilización en la vida adulta, en enfermedades crónicas como la psoriasis ${ }^{34,35)}$.

Darné y colaboradores ${ }^{\left({ }^{36}\right)}$ describieron la utilización de máscaras de disfraz adaptando el tratamiento y minimizando los riesgos y efectos secundarios (eritema, xerosis, prurito) causados por la exposición de la piel facial sin lesiones. En un estudio retrospectivo que comparó adultos y niños, Magdaleno-Tapial y colaboradores no encontraron diferencias estadísticamente significativas en cuanto a dosis, duración o número de sesiones, pero sí en la mayor adherencia en el grupo de niños ${ }^{(2)}$.

\section{OBJETIVOS}

Con el fin de describir y compartir nuestros conocimientos relacionados con la utilización de la fototerapia en niños, se realizó este trabajo que hará énfasis 
Tabla 1. Mecanismos de acción de la fototerapia

\begin{tabular}{|c|c|c|}
\hline Patología & Tipo de fototerapia & Mecanismo de acción \\
\hline Vitíligo & UVB NB/PUVA tópica & $\begin{array}{l}\text { Regulación positiva, aumento de } \\
\text { la melanogénesis y migración de } \\
\text { melanocitos en los folículos } \\
\text { pilosos }\end{array}$ \\
\hline Psoriasis & UVB NB/PUVA & $\begin{array}{l}\text { Aumento de la interleucina } 10 \text { y de } \\
\text { las prostaglandinas con alteración } \\
\text { de la activación de las células } \\
\text { dendríticas y del equilibrio del } \\
\text { linfocito T efector/regulador. } \\
\text { Disminución de la proliferación de } \\
\text { queratinocitos. Supresión de la } \\
\text { expresión de las moléculas de } \\
\text { adhesión intercelular y de los } \\
\text { mediadores inmunosupresores } \\
\text { solubles. }\end{array}$ \\
\hline Dermatitis atópica & UVB NB/UVA1 & Véase Psoriasis \\
\hline Morfea & UVB NB/UVA1 & $\begin{array}{l}\text { Aumento de la colagenasa } 1 \text { por } \\
\text { los fibroblastos, aumento de las } \\
\text { interleucinas } 1 \text { y } 6 \text { con aumento en } \\
\text { la producción de metaloproteinasa } \\
\text { de la matriz. }\end{array}$ \\
\hline Micosis fungoide & UVB NB/PUVA & Apoptosis de linfocitos $\mathrm{T}$. \\
\hline
\end{tabular}

en aspectos que difieren de lo realizado en adultos en cuanto a indicaciones, tipo de fototerapia, tiempo de tratamiento, cuidados, requisitos, entre otros. Se tomaron datos de las guías y las publicaciones existentes (3), sumado a nuestra experiencia de manejo en los últimos 10 años ${ }^{(29)}$. Nuestra intención es transmitir los aspectos relevantes del uso de fototerapia en niños, rescatar su utilidad en la edad pediátrica, sus beneficios y desventajas frente a otros tratamientos sistémicos. La medicina basada en la evidencia en pediatría, a diferencia de la enfocada en los adultos, está limitada por los escasos estudios realizados en niños que evalúan la eficacia y la seguridad de la fototerapia en población pediátrica $^{(2,3,25,37)}$.

\section{¿CUÁNDO ESTÁ INDICADA?}

- Afección de más del 3\%-10\% de la superficie corporal.

- Falta de respuesta a la terapia tópica o limitaciones para su uso.

- Distribución generalizada.

- Gran impacto emocional o discapacidad.

\section{¿TIENE CONTRAINDICACIONES?}

Para empezar, una de las primeras cosas de las que nos debemos asegurar en la primera consulta con los niños 
es que tengan la capacidad de tolerar o permanecer quietos dentro de la cabina. En pacientes de menor edad o que presenten movilidad limitada o patologías con pérdida del equilibrio se pueden utilizar paneles abiertos y la ayuda de los padres o tutores. Las tecnologías de excimer, usadas en adultos en caso de lesiones escasas localizadas, pueden ser utilizadas en adolescentes o en niños con dificultad técnica si no pueden permanecer quietos ${ }^{(6)}$.

Consideraciones generales:

- Enfermedad o antecedente de neoplasias de la piel (melanoma y no melanoma).

- Tratamientos inmunosupresores concomitantes.

- Fotosensibilidad (autoinmunidad/drogas).

- En relación con los psoralenos orales (afaquia, embarazo, lactancia, disfunción hepática, cataratas).

\section{¿CUÁL ELEGIR?}

La modalidad de tratamiento dependerá de la patología, la profundidad de las lesiones, la edad, las áreas corporales afectadas, la zona de vivienda, entre otros (20, 22, 26, 30, 35, 38-43).

\section{UVB NB}

Es la más utilizada en niños con afecciones que comprometen la epidermis, comola psoriasis, el vitíligo, la dermatitis atópica, la alopecia areata ${ }^{(44)}$, el liquen plano ${ }^{(45)}$, la PLEVA, la PLC y la hipomelanosis macular progresiva que no responde a peróxido de benzoílo $(46,47)$. Es fácil de implementar, no requiere ingesta de psoralenos, protección ocular, ni controles periódicos de laboratorio, lo que aumenta la adherencia. Puede realizarse en pacientes en edad reproductiva o embarazadas, ya que no afecta al feto ni requiere controles. Es útil como terapia de mantenimiento (1-2 veces/semana) posterior al tratamiento de la MF con PUVA. En dermatitis atópica tiene efecto sobre la microbiota de la piel reflejado en la disminución de Staphylococcus aureus ${ }^{(48)}$.

\section{PUVA}

Indicada en mayores de 12 años, requiere controles de laboratorio previos y control oftalmológico (cristalino y conjuntiva) para evaluar la presencia de cataratas y cambios conjuntivales.
En un estudio prospectivo de 25 años (1993-2004), Malanos-Stern no encontró un incremento del riesgo usando protección ocular ${ }^{(49)}$. La ingesta de psoraleno debe ser dos horas antes de la exposición a UVA (con alimento) y se recomienda el uso de anteojos con protección UV el resto del día y no estar al aire libre. Excepcionalmente puede utilizarse en menores de 12 años cuando la patología lo amerite o no haya respuesta a la UVB NB. Tiene la ventaja sobre la UVB NB de que alcanza períodos de remisión más prolongados.

\section{PUVA tópico}

Psoraleno en cremas, ungüentos y lociones con 8 metoxipsoraleno (8-MOP) al 0,01\%-0,001\%, 15 a 30 minutos previos a la exposición a UVA. Psoriasis en placa o limitada y palmoplantar, vitíligo localizado focal, generalizado sin respuesta a UVB, con menos del 10\%-20\% de superficie corporal. La distribución no uniforme del producto puede inducir eritema fototóxico y parches de hiperpigmentación incluso perilesional.

\section{PUVA baño}

Utilizado en psoriasis, constituye un tratamiento complejo de realizar, pues requiere infraestructura adecuada. 8-MOP 2,5 mg/L, diluido en $36 \mathrm{~mL}$ de una solución alcohólica de 8 -MOP al $1 \%$ en $140 \mathrm{~L}$ de agua entre $37^{\circ} \mathrm{C}-42^{\circ} \mathrm{C}$. La zona afectada debe sumergirse durante 20 minutos y después secarse y exponerse a UVA parcial o total ${ }^{(50)}$.

\section{UVA1}

En enfermedades que comprometen la dermis profunda, como la esclerodermia localizada en placas (morfea) versus lineal, o en dermatitis atópica moderada o sin respuesta a la UVB NB. Tiene la desventaja de requerir mayor tiempo de exposición, lo que dificulta la tolerancia por el exceso de calor ya que pocos aparatos cuentan con una ventilación adecuada por lo que es necesario adaptar sistemas de enfriamiento adicionales o hacer pausas durante las sesiones de fototerapia.

\section{¿CÓMO SE REALIZA?}

Se deben consensuar previamente con la familia los aspectos inherentes al tratamiento, para asegurar la comprensión y el cumplimiento. Para esto se requiere: 
1. Paciente esté acompañado por un mayor de edad.

2. Firma del consentimiento o asentimiento una vez comprendida la explicación.

3. El niño y sus tutores conozcan previamente los aparatos, para generar confianza y tranquilidad; en caso contrario, postergue el tratamiento.

4. Tratamiento trisemanal con intervalos no menores de 26 horas entre sesiones. Si la evolución es favorable, se inicia un esquema descendente de 2 veces por semana; en algunos casos se realizan 1 a 2 sesiones semanales como terapia de mantenimiento. Estas frecuencias, dosis e incrementos dependen del fototipo o de la susceptibilidad y tipo de patología cutánea que requiera tratamiento. En niños, el cálculo de dosis eritematosas mínimas resulta poco práctico de realizar, por lo cual se hace basado en el fototipo.

5. En pacientes que vivan en un domicilio alejado, aproximadamente a $300 \mathrm{~km}$ del centro, o de difícil acceso se puede realizar el tratamiento domiciliario con supervisión y controles presenciales cada 2 meses o este puede llevarse a cabo en el centro de fototerapia intensiva 4 veces por semana, con opción de uso de equipos en el domicilio. La ley de accesibilidad a los aparatos en domicilio varía de acuerdo con cada país; en Argentina, la Administración Nacional de Alimentos y Medicamentos (ANMAT) permite su comercialización para uso profesional.

6. Es necesario que el paciente permanezca inmóvil frente o dentro del aparato para evitar accidentes.

7. Advertir a los pacientes y padres que la variabilidad en la respuesta al tratamiento depende de la patología y del sitio corporal afectado, para evitar falsas expectativas y falla en la adherencia.

8. En casos de vitíligo, advertir la pobre respuesta en zona acrales y que durante el tratamiento la piel puede tornarse tricolor (piel normal, vitíligo y máculas hipercrómicas de re pigmentación). La variabilidad en esta respuesta ha sido estudiada por Nicolaidou y colaboradores ${ }^{(51)}$ y por Brazzelli y colaboradores ${ }^{(52)}$.

9. Exponer solo las zonas afectadas y en el caso de párpados valorar el uso de anteojos. Nunca exponer los genitales masculinos. Para mejorar la adherencia al tratamiento se implementó el uso de máscaras y disfraces para evitar la exposición a RUV y hacerlo entretenido (figuras 1 a 4) ${ }^{(36)}$.

10. Siguiendo el consentimiento informado, tomar iconografías de inicio y en cada control para registrar la evolución, lo cual permitirá objetivar la respuesta.
11. Las dosis se calculan dependiendo del fototipo de piel (tabla 2). Los incrementos se realizarán aproximadamente en un 10\%-15\% de la dosis previa y cada dos sesiones ${ }^{(3)}$.

12. Hacer énfasis en la importancia de los controles y para evaluar la efectividad de los tratamientos. Reprogramar las dosis por ausencias puede derivar en fallas en la respuesta completa. Las respuestas parciales pueden deberse a las características del propio paciente, como si son respondedores (rápidos o lentos), hay falla terapéutica, las terapias de mantenimiento son irregulares, hay recaídas y recurrencias.

13. Los efectos esperados son leves y transitorios y rara vez llevan a suspender el tratamiento: el eritema, la xerodermia y el prurito disminuyen con fotoprotección e hidratación posterior a la sesión. Los efectos adversos relacionados con la fototerapia PUVA suelen ser molestias gastrointestinales causadas por el psoraleno, que se previenen tomando este último con el alimento. A largo plazo puede presentarse carcinogénesis ${ }^{(1)}$.

14. Recomendar la debida información sobre el uso de medicamentos fotosensibles, en especial los grupos anticonvulsivantes, antidepresivos y antiinflamatorios no esteroideos (AINE). Evitar la automedicación.

15. En las primeras 10 sesiones se evalúa la tolerancia, el cumplimiento, la presencia de efectos adversos, la falta de interés, la dificultad de acceso al centro y se refuerzan todas las pautas e indicaciones. La mejoría varía de acuerdo con la patología. En niños, la psoriasis en placas puede mostrar mejoría inicial con 5-6 sesiones. En los casos de vitíligo se requieren cerca de 30-40 sesiones para observar el inicio de la re pigmentación. Es importante advertir lo anterior para no generar falsas expectativas y abandonos prematuros.

16. En caso de eritema sintomático, enrojecimiento, ardor y molestia, se recomienda la suspensión transitoria de la fototerapia y repasar las posibles causas como, por ejemplo, el no uso de protección adecuada, la exposición a radiación al aire libre, el uso de productos con alquitrán, como el champú, el aceite mineral, entre otros ${ }^{\left.{ }^{53}\right)}$. Deberán usarse emolientes y en ocasiones corticoides de baja potencia cada 12 horas durante 5 a 7 días. En caso de pérdida de sesiones, se propone el esquema planteado por Carrascosa y colaboradores ${ }^{(3)}$ : pérdida de 1 sesión: mantener la dosis previa; pérdida de 1 semana: disminuir la dosis en un $25 \%$; pérdida de 2-3 semanas: disminuir la dosis en un 50\%; y 

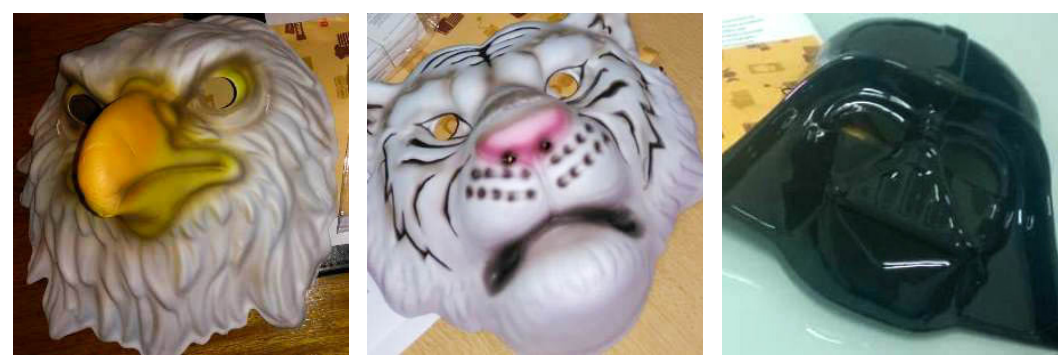

Figura 1, 2, 3. Máscara para protección de la cara en los niños durante la fototerapia. Estas máscaras están a la venta en casas de cotillón para fiestas. Se deben adaptar con anteojos si se quiere cubrir párpados. La foto fué tomada por la autora para acompañar este escrito.

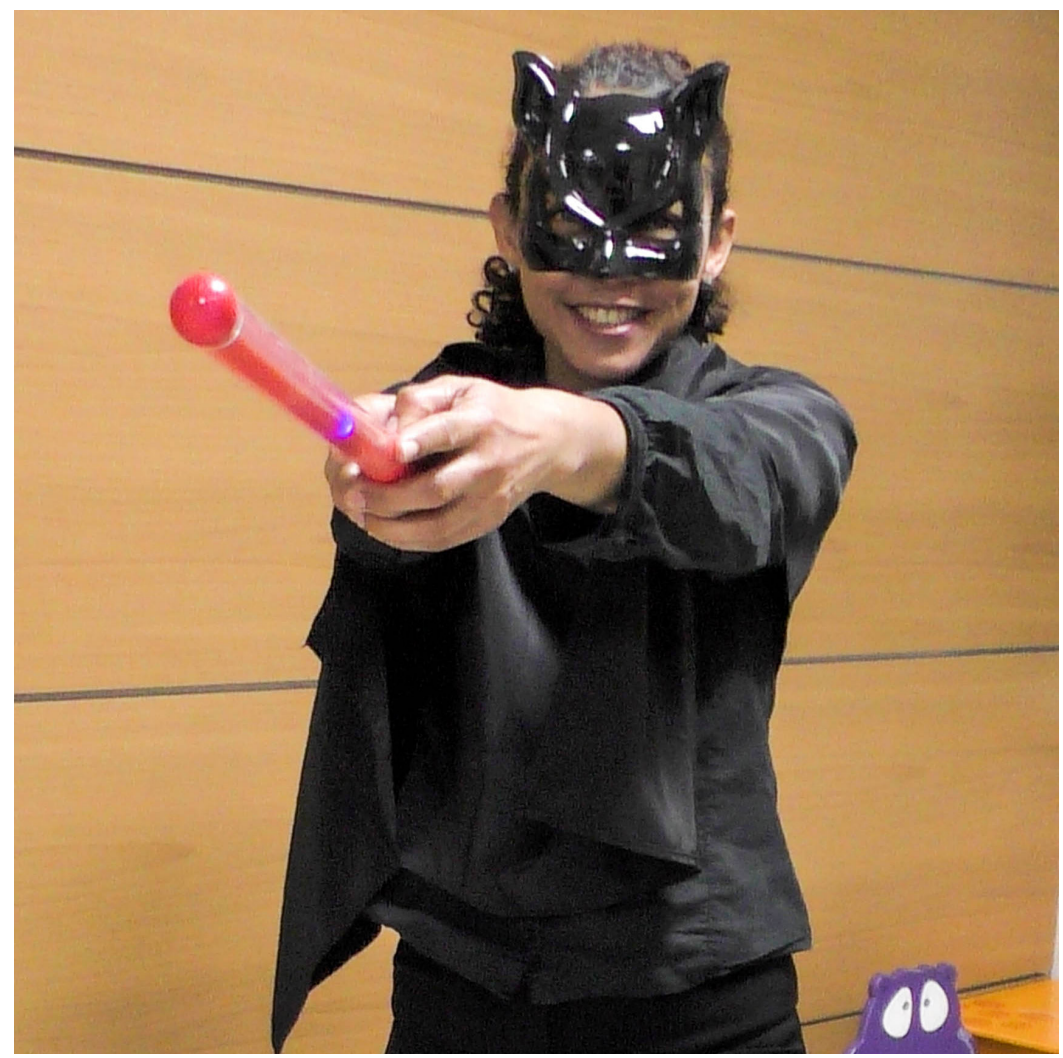

Figura 4. Máscara para protección de la cara en los niños durante la fototerapia. En compañía del médico tratante y la familia, es importante que el niño sienta confianza en su tratamiento con una aproximación lúdica al mismo. Estas máscaras están a la venta en casas de cotillón para fiestas. Se deben adaptar con anteojos si se quiere cubrir párpados. En esta foto de la autora como una demostración de su uso, tomada para acompañar este escrito. 
Tabla 2. Tratamiento

\begin{tabular}{|c|c|c|c|c|c|c|}
\hline Patología & $\begin{array}{c}\text { Tipo de } \\
\text { fototerapia }\end{array}$ & $\begin{array}{l}\text { Dosis de inicio } \\
\qquad / \mathrm{cm}_{2}\end{array}$ & $\begin{array}{l}\text { Inicio de } \\
\text { respuesta } \\
\text { sesión n. }\end{array}$ & $\begin{array}{c}\text { Evidencia } \\
\text { clínica de } \\
\text { mejoría }\end{array}$ & $\begin{array}{l}\text { Dosis máxima } \\
\text { aproximada } \\
\text { requerida } \\
\mathrm{J} / \mathrm{cm}^{2^{*}}\end{array}$ & $\begin{array}{l}\text { Número de } \\
\text { sesiones } \\
\text { aproximado/ } \\
\text { ciclo }\end{array}$ \\
\hline Vitíligo & $\begin{array}{l}\text { UVB NB } \\
\text { PUVA tópica }{ }^{\ddagger}\end{array}$ & $0,145 \mathrm{~J} / \mathrm{cm}^{2}$ & 40 & $\begin{array}{l}\text { Pigmentación } \\
\text { perifolicular }\end{array}$ & $1,040 \mathrm{~J} / \mathrm{cm}^{2}$ & $50-80$ \\
\hline Psoriasis & $\begin{array}{l}\text { UVB NB } \\
\text { PUVA }^{\dagger} \\
\text { PUVA tópico }\end{array}$ & $\begin{array}{c}0,145 \cdot 0,175 \\
\mathrm{~J} / \mathrm{cm}^{2}\end{array}$ & $5-10$ & $\begin{array}{l}\text { Mejoría del } \\
\text { prurito, } \\
\text { disminución de } \\
\text { las lesiones }\end{array}$ & $2,000 \mathrm{~J} / \mathrm{cm}^{2}$ & $10-40$ \\
\hline $\begin{array}{l}\text { Dermatitis } \\
\text { atópica }\end{array}$ & UVB NB & $\begin{array}{c}0,145-0,175 \\
\mathrm{~J} / \mathrm{cm}^{2}\end{array}$ & $10-20$ & $\begin{array}{l}\text { Mejoría del } \\
\text { prurito, y } \\
\text { disminución de } \\
\text { la microbiota y } \\
\text { la colonización } \\
\text { bacteriana, que } \\
\text { actúa como } \\
\text { superantígeno. }\end{array}$ & $1,196 \mathrm{~J} / \mathrm{cm}^{2}$ & $30-60$ \\
\hline Morfea & UVB NB & $\begin{array}{c}0,145-0,175 \\
\mathrm{~J} / \mathrm{cm}^{2}\end{array}$ & $30-40$ & $\begin{array}{l}\text { Disminución de } \\
\text { la esclerosis } \\
\text { cutánea, } \\
\text { aumento de su } \\
\text { laxitud }\end{array}$ & $20-30 \mathrm{~J} / \mathrm{cm}^{2}$ & $50-90$ \\
\hline $\begin{array}{l}\text { Micosis } \\
\text { fungoide } \\
\text { hipopigmen- } \\
\text { tada }\end{array}$ & UVB NB & $\begin{array}{c}0,145-0,175 \\
\mathrm{~J} / \mathrm{cm}^{2} \\
1 \mathrm{~J} / \mathrm{cm}^{2}+ \\
\text { psoraleno }\end{array}$ & $30-40$ & $\begin{array}{l}\text { Disminución, } \\
\text { aclaramiento y } \\
\text { desaparición de } \\
\text { las lesiones de } \\
\text { la piel en niños }\end{array}$ & $15 \mathrm{~J} / \mathrm{cm}^{2}$ & $80-110$ \\
\hline
\end{tabular}

* Joules/cm²; †psoraleno: 8 metoxipsoraleno 0,4 mg/kg/dosis; ‡ psoraleno: 0,001\%. Nota: tratamiento trisemanal. 


\section{Puntos clave}

- Los pacientes pediátricos no son adultos en miniatura, por lo que requieren conocimiento del manejo pediátrico y dermatológico.

- Lograr la adherencia es un pilar muy importante para el cumplimiento y éxito del tratamiento.

- Se debe incentivar la participación y acompañamiento de la familia como parte del tratamiento.

pérdida de más de 3 semanas: reiniciar el tratamiento.

17. El tiempo de respuesta varía según la patología (véase la tabla 2). Teniendo en cuenta que son niños, el tratamiento debe flexibilizarse contemplando los períodos vacacionales y las actividades escolares y deportivas.

18. Es importante hidratar la piel con emulsiones, lo que favorece la respuesta a la RUV en las enfermedades eritematoescamosas y mejora el prurito.

19. No retirar efectivamente las sustancias tópicas antes de ingresar al aparato puede aumentar el efecto de la RUV o generar fotosensibilidad (alquitrán de hulla o aceite mineral). Otras sustancias, como el ácido salicílico y el calcipotriol, se pueden inactivar.

20. El paciente debe avisar al personal si está bajo tratamiento farmacológico o presenta fiebre, brotes $\mathrm{u}$ otros síntomas previos al inicio de la sesión.

21. Se deben tener en cuenta otros aspectos como la edad, el estado emocional, los factores desencadenantes, el grado escolar y la disrupción familiar. Siempre se debe incluir a la familia como parte activa del tratamiento.

22. Es importante reforzar las medidas de fotoeducación: evitar las exposiciones innecesarias a la radiación ultravioleta, la exposición de 11 a 17 horas y el uso de protección solar con un factor de igual o mayor a 50 FPS; promover el uso de gorros y ropa, así como los deportes a la sombra para evitar el fotodaño, ya que por tratarse de patologías crónicas es probable que requieran fototerapia a futuro.

\section{¿QUÉ CANTIDAD DE SESIONES SE PUEDEN TOMAR? ¿HAY RIESGO DE CARCINOGÉNESIS?}

A 40 años de su implementación, el límite propuesto para la PUVA en adultos es de 250 sesiones ${ }^{(1,54)}$. En UVB no hay un límite establecido y los trabajos mencionan un promedio de 30 a 40 sesiones para mejorar las lesiones. La cantidad de sesiones que deban realizarse dependerá de la respuesta obtenida, la tolerancia, la patología, la edad, el segmento corporal y la regularidad del cumplimiento (véase la tabla 2). A la fecha, no existe evidencia de carcinogénesis por UVB NB. Se debe tener claridad de los antecedentes de tratamientos de fototerapia, tipo, cantidad de sesiones y dosis total acumulada, parámetros útiles y necesarios para definir los tratamientos a futuro. Lee y colaboradores, en una revisión de publicaciones realizadas entre 1966 y 2002, que incluyeron cerca de 3400 participantes, no encontraron un incremento en el riesgo de cáncer por UVB NB ${ }^{(55)}$.

\section{¿SE PUEDE ROTAR O COMBINAR CON OTROS TRATAMIENTOS?}

Sí, se puede combinar con tópicos, aunque teniendo precaución con aquellos que generan irritación si contienen alquitrán, en caso de usarlos, deben ser retirados antes del ingreso a la cámara, para evitar reacción de 
fotosensibilidad y/o quemaduras. El calcipotriol o el ácido salicílico, que pueden inactivarse, se deben usar después de la sesión, luego de hidratar la piel. Los sistémicos como el metotrexato y los retinoides pueden ser administrados con UVB NB o con PUVA, prefiriendo UVB NB. En casos de morfea se pueden combinar UVA1 con calcipotriol ${ }^{(56)}$ o en caso de lesiones activas, corticoide en pulsos más metotrexato (este último tiene un efecto de acción más tardío). Las dosis y tiempos necesarios suelen ser menores por el efecto sinérgico de la combinación y hacen de la fototerapia un ahorrador de tratamiento ${ }^{(3)}$. Los controles serán los habituales para cada sistémico. Como menciona Stern ${ }^{(1)}$, se debe considerar el riesgo/beneficio de la inmunosupresión y elegir el mejor tratamiento, acompañado de un seguimiento a largo plazo.

En el caso de los inmunomoduladores tópicos ${ }^{(57)}$ (tacrolimus, pimecrolimus), se deben colocar en días alternos a la sesión de fototerapia, ya que pueden resultar irritantes y potenciar la inmunosupresión.

\section{¿LOS PACIENTES PUEDEN REALIZAR ACTIVIDADES ESCOLARES Y DEPORTIVAS?}

Esta es una pregunta frecuente de los padres. Buscar flexibilidad es la regla y que el tratamiento no interfiera. En actividades al aire libre, intensificar la fotoprotección evitando las exposiciones en los horarios de mayor incidencia de la RUV.

\section{COMENTARIOS FINALES}

- La fototerapia UVB NB es de elección en niños por su facilidad de implementación y efectividad, con pocos efectos adversos.

- El manejo en niños debe incluir el acompañamiento de la familia y los aspectos escolares, sociales y emocionales.

- Se debe elegir el mejor tratamiento para cada caso en particular, sin incurrir en un tratamiento deficiente.

- La fototerapia debe utilizarse con cautela y en el tiempo necesario y suficiente para controlar la enfermedad y mejorar la calidad de vida.

- El seguimiento a largo plazo permite acompañar y pesquisar la aparición de efectos adversos.
- $\quad$ El uso de fotoprotectores y de barreras físicas (máscaras) aseguran una mejor tolerancia, adherencia y cumplimiento.

\section{REFERENCIAS}

1. Stern RS, PUVA Follow-Up Study. The risk of squamous cell and basal cell cancer associated with psoralen and ultraviolet A therapy: a 30year prospective study. J Am Acad Dermatol [Internet]. 2012 Apr;66(4):553-62. Available from: http://dx.doi.org/10.1016/j.jaad.2011.04.004

2. Magdaleno-Tapial J, Ortiz-Salvador JM, Valenzuela-Oñate C, Marí-Cornejo P, Esteve-Martínez A, Pérez-Ferriols A. Comparison of Phototherapy in Pediatric and Adult Patients. Actas Dermosifiliogr [Internet]. 2019 May 24; Available from: http://dx.doi.org/10.1016/j.ad.2019.03.012

3. Carrascosa JM, Gardeazábal J, Pérez-Ferriols A, Alomar A, Manrique P, Jones-Caballero M, Lecha M, Aguilera J, De La Cuadra J. [Consensus document on phototherapy: PUVA therapy and narrow-band UVB therapy]. Actas Dermosifiliogr [Internet]. 2005 Dec;96(10):635-58. Available from: http://dx.doi.org/10.1016/sooo1-7310(05)73153-7

4. Welsh O, Herz-Ruelas ME, Gómez M, OcampoCandiani J. Therapeutic evaluation of UVB-targeted phototherapy in vitiligo that affects less than $10 \%$ of the body surface area. Int J Dermatol [Internet]. 2009 May;48(5):529-34. Available from: http://dx.doi.org/10.1111/j.1365-4632.2009.03928.x

5. Ohtsuki A, Hasegawa T, Komiyama E, Takagi A, Kawasaki J, Ikeda S. 308-nm Excimer Lamp for the Treatment of Alopecia Areata: Clinical Trial on 16 Cases. Indian J Dermatol [Internet]. 2013 Jul;58(4):326. Available from: http://dx.doi.org/10.4103/o019-5154.113954

6. Abrouk M, Levin E, Brodsky M, Gandy JR, Nakamura M, Zhu TH, Farahnik B, Buthani T. Excimer laser for the treatment of psoriasis: safety, efficacy, and patient acceptability. Psoriasis (Auckl) [Internet]. 2016 Dec 12;6:165-73. Available from: http://dx.doi.org/10.2147/PTT.S105047

7. Tartar D, Bhutani T, Huynh M, Berger T, Koo J. Update on the immunological mechanism of action behind phototherapy. J Drugs Dermatol [Internet]. 2014 May;13(5):564-8. Available from: https://www.ncbi.nlm.nih.gov/pubmed/24809879

8. Kerr AC, Ferguson J, Attili SK, Beattie PE, Coleman AJ, Dawe RS, Eberlein B, Goulden V, Ibbotson SH, 
Menage Hdu P, Moseley H, Novakovic L et al. Ultraviolet A1 phototherapy: a British Photodermatology Group workshop report. Clin Exp Dermatol [Internet]. 2012 Apr;37(3):219-26. Available from: http://dx.doi.org/10.1111/j.1365-2230.2011.04256.x

9. Tuchinda C, Kerr HA, Taylor CR, Jacobe H, Bergamo BM, Elmets C, Rivard J, Lim HW. UVA1 phototherapy for cutaneous diseases: an experience of 92 cases in the United States. Photodermatol Photoimmunol Photomed [Internet]. 2006 Oct;22(5):247-53. Available from: http://dx.doi.org/10.1111/j.1600-0781.2006.00245.x

10. Welsh O. Phototherapy for alopecia areata. Clin Dermatol [Internet]. 2016 Sep;34(5):628-32. Available from: http://dx.doi.org/10.1016/j.clindermatol.2016.05. 014

11. Dainichi T, Kabashima K. Alopecia areata: What's new in epidemiology, pathogenesis, diagnosis, and therapeutic options? J Dermatol Sci [Internet]. $2017 \mathrm{Apr} ; 86(1): 3-12$. Available from: http://dx.doi.org/10.1016/j.jdermsci.2016.10.004

12. Mohammad TF, Al-Jamal M, Hamzavi IH, Harris JE, Leone G, Cabrera R, Lim HW, Pandya AG, Esmat SM. The Vitiligo Working Group recommendations for narrowband ultraviolet B light phototherapy treatment of vitiligo [Internet]. Vol. 76, Journal of the American Academy of Dermatology. 2017. p. 879-88. Available from: http://dx.doi.org/10.1016/j.jaad.2016.12.041

13. Hodak E, Pavlovsky L. Phototherapy of Mycosis Fungoides [Internet]. Vol. 33, Dermatologic Clinics. 2015. p. 697-702. Available from: http://dx.doi.org/10.1016/j.det.2015.05.005

14. Meduri NB, Bhavani Meduri N, Vandergriff $\mathrm{T}$, Rasmussen $\mathrm{H}$, Jacobe $\mathrm{H}$. Phototherapy in the management of atopic dermatitis: a systematic review [Internet]. Vol. 23, Photodermatology, Photoimmunology \& Photomedicine. 2007. p. 106-12. Available from: http://dx.doi.org/10.1111/j.1600-0781.2007.00291.x

15. Kreuter A, Hyun J, Stücker M, Sommer A, Altmeyer P, Gambichler T. A randomized controlled study of low-dose UVA1, medium-dose UVA1, and narrowband UVB phototherapy in the treatment of localized scleroderma [Internet]. Vol. 54, Journal of the American Academy of Dermatology. 2006. p. 440-7. Available from: http://dx.doi.org/10.1016/j.jaad.2005.11.1063

16. González-Ardila C, Londoño-García A, Cortés-Correa C, Asociación Colombiana de Dermatología y Cirugía Dermatológica - AsoColDerma, Grupo
Colombiano de Psoriasis y Artritis Psoriásica ColPsor. Editores. Guías basadas en la evidencia para el manejo de la psoriasis en Colombia. 2018. S111

17. Dawe RS. Ultraviolet A1 phototherapy. BrJ Dermatol [Internet]. 2003 Apr;148(4):626-37. Available from: http://dx.doi.org/10.1046/j.1365-2133.2003.05261.x

18. Coronel-Pérez IM, Carrizosa-Esquivel AM, Camacho-Martínez F. [Narrow band UVB therapy in early stage mycosis fungoides. A study of 23 patients]. Actas Dermosifiliogr [Internet]. 2007 May;98(4):259-64. Available from: https://www.ncbi.nlm.nih.gov/pubmed/17506957

19. Grupo Argentino de psoriasis. Sociedad Argentina de Dermatología. Guia de tratamiento. Consenso Nacional de Psoriasis,. 2018;9,10.

20. Eustace K, Dolman S, Alsharqi A, Sharpe G, Parslew R.UseofPhototherapyinChildren.PediatrDermatol [Internet]. 2017 Mar;34(2):150-5. Available from: http://dx.doi.org/10.1111/pde.13072

21. Ersoy-Evans S, Altaykan A, Sahin S, Kölemen F. Phototherapy in childhood. Pediatr Dermatol [Internet]. 2008 Nov;25(6):599-605. Available from: http://dx.doi.org/10.1111/j.1525-1470.2008.00773.x

22. Crall CS, Rork JF, Delano S, Huang JT. Phototherapy in children: Considerations and indications. Clin Dermatol [Internet]. 2016 Sep;34(5):633-9. Available from: http://dx.doi.org/10.1016/j.clindermatol.2016.05. 018

23. Jury CS, McHenry P, Burden AD, Lever R, Bilsland D. Narrowband ultraviolet B (UVB) phototherapy in children [Internet]. Vol. 31, Clinical and ExperimentalDermatology.2006.p.196-9.Availablefrom: http://dx.doi.org/10.1111/j.1365-2230.2006.02061.x

24. Pasic A, Ceovic R, Lipozencic J, Husar K, Susic $\mathrm{SM}$, Skerlev $\mathrm{M}$, et al. Phototherapy in Pediatric Patients [Internet]. Vol. 20, Pediatric Dermatology. 2003. p. 71-7. Available from: http://dx.doi.org/10.1046/j.1525-1470.2003.03016.x

25. Koo J, Nakamura M. Clinical Cases in Phototherapy [Internet]. 2017. Available from: http://dx.doi.org/10.1007/978-3-319-51599-1

26. Koh MJ-A, Mok Z-R, Chong W-S. Phototherapy for the treatment of vitiligo in Asian children. Pediatr Dermatol [Internet]. 2015 Mar;32(2):192-7. Available from: http://dx.doi.org/10.1111/pde.12506

27. Ubogui J, Saposnik M. Uso de la fototerapia ultravioleta en pediatría. In: Larralde M, Abad E, Luna P, editores. Dermatología pediátrica 2da edición. 2010. p. 651-4. 
28. Cervini AB, Torres-Huamani AN, Sanchez-LaRosa C, Galluzzo L, Solernou V, Digiorge J, Rubio P. Micosis fungoide. Experiencia en un hospital pediátrico [Internet]. Vol. 108, Actas DermoSifiliográficas. 2017. p. 564-70. Available from: http://dx.doi.org/10.1016/j.ad.2017.01.008

29. Ibáñez-Gómez D, Moumdjian H, De La Sota R, Chuit R, Vedia Y, Garcia-Pazos ML, Saposnik M, Ubogui J. Phototherapy in children: 10 years of experience [Internet]. Vol.81, Journal of the American Academy of Dermatology. 2019. p. AB103. Available from: http://dx.doi.org/10.1016/j.jaad.2019.06.397

30. Tan E, Lim D, Rademaker M. Narrowband UVB phototherapy in children: A New Zealand experience. Australas J Dermatol [Internet]. 2010 Nov;51(4):268-73. Available from: http://dx.doi.org/10.1111/j.1440-0960.2010.00701.x

31. Percivalle S, Piccinno $\mathrm{R}$, Caccialanza $\mathrm{M}$, Forti S. Narrowband ultraviolet B phototherapy in childhood vitiligo: evaluation of results in 28 patients. Pediatr Dermatol [Internet]. 2012 Mar;29(2):160-5. Available from: http://dx.doi.org/10.1111/j.1525-1470.2011.01683.x

32. Valejo Coelho MM, Matos TR, Apetato M. The dark side of the light: mechanisms of photocarcinogenesis. Clin Dermatol [Internet]. 2016 Sep;34(5):563-70. Available from: http://dx.doi.org/10.1016/j.clindermatol.2016.05. 022

33. Coelho MMV, Apetato M. The dark side of the light: Phototherapy adverse effects [Internet]. Vol.34, ClinicsinDermatology.2016.p.556-62. Availablefrom: http://dx.doi.org/10.1016/j.clindermatol.2016.05. 005

34. Jain VK, Aggarwal K, Jain K, Bansal A. Narrowband UV-B phototherapy in childhood psoriasis [Internet]. Vol. 46, International Journal of Dermatology. 2007. p. 320-2. Available from: http://dx.doi.org/10.1111/j.1365-4632.2007.03148.x

35. Zamberk P, Velázquez D, Campos M, Hernanz JM, Lázaro P. Paediatric psoriasis - narrowband UVB treatment [Internet]. Vol. 24, Journal of the European Academy of Dermatology and Venereology. 2010. p. 415-9. Available from: http://dx.doi.org/10.1111/j.1468-3083.2009.03425.x

36. Darné S, Leech SN, Taylor AEM. Narrowband ultraviolet $\mathrm{B}$ phototherapy in children with moderate-to-severe eczema: a comparative cohort study. $\mathrm{Br} \mathrm{J}$ Dermatol [Internet]. 2014 Jan;170(1):150-6. Available from: http://dx.doi.org/10.1111/bjd.12580

37. Escudero M del M, del Mar Escudero M, Es- calas J. Seguridad y utilidad de la fototerapia con rayos ultravioleta $B$ de banda estrecha en la edad pediátrica [Internet]. Vol. 30, Piel. 2015. p. 619-20. Available from: http://dx.doi.org/10.1016/j.piel.2015.07.006

38. Koo J, Nakamura M. Phototherapy for the Pediatric Population [Internet]. Clinical Cases in Phototherapy. 2017. p. 139-43. Available from: http://dx.doi.org/10.1007/978-3-319-51599-1_22

39. Pugashetti R, Koo J. Phototherapy in pediatric patients: choosing the appropriate treatment option. Semin Cutan Med Surg [Internet]. 2010 Jun;29(2):115-20. Available from: http://dx.doi.org/10.1016/j.sder.2010.03.006

40. Mok Z-R, Koh MJ-A, Chong W-S. Is phototherapy useful in the treatment of atopic dermatitis in asian children? A 5-year report from singapore. Pediatr Dermatol [Internet]. 2014 Nov;31(6):698-702. Available from: http://dx.doi.org/10.1111/pde.12405

41. Laws PM, Shear NH, Pope E. Childhood mycosis fungoides: experience of 28 patients and response to phototherapy. Pediatr Dermatol [Internet]. 2014 Jul;31(4):459-64. Available from: http://dx.doi.org/10.1111/pde.12338

42. Koh MJ-A, -A. Koh MJ, -S. Chong W. Narrow-band ultraviolet B phototherapy for mycosis fungoides in children [Internet]. Vol. 39, Clinical and ExperimentalDermatology.2014.p.474-8. Availablefrom: http://dx.doi.org/10.1111/ced.12364

43. Pavlovsky M, Baum S, Shpiro D, Pavlovsky L, Pavlotsky F. Narrow band UVB: is it effective and safe for paediatric psoriasis and atopic dermatitis? J Eur Acad Dermatol Venereol [Internet]. 2011 Jun;25(6):727-9. Available from: http://dx.doi.org/10.1111/j.1468-3083.2010.03832.x

44. Bayramgürler D, Demirsoy EO, Aktürk AŞ, Kiran R. Narrowband ultraviolet B phototherapy for alopecia areata [Internet]. Vol. 27, Photodermatology, Photoimmunology \& Photomedicine. 2011. p. 325-7. Available from: http://dx.doi.org/10.1111/j.1600-0781.2011.00612.x

45. Taneja A, Taylor CR. Narrow-band UVB for lichen planus treatment [Internet]. Vol. 41, International Journal of Dermatology. 2002. p. 282-3. Available from: http://dx.doi.org/10.1046/j.1365-4362.2002.01499.x

46. Sim JH, Lee DJ, Lee JS, Kim YC. Comparison of the clinical efficacy of NBUVB and NBUVB with benzoyl peroxide/clindamycin in progressive macular hypomelanosis [Internet]. Vol. 25, Journal of the European Academy of Dermatology and 
Venereology. 2011. p. 1318-23. Available from: http://dx.doi.org/10.1111/j.1468-3083.2011.03980.x

47. Montero LC, Belinchón I, Toledo F, Betlloch I. Progressive macular hypomelanosis, excellent response with narrow-band ultraviolet B phototherapy [Internet]. Vol. 27, Photodermatology, Photoimmunology \& Photomedicine. 2011. p. 162-3. Available from: http://dx.doi.org/10.1111/j.1600-0781.2011.00583.x

48. Dotterud LK, Wilsgaard T, Vorland LH, Falk ES. The effect of UVB radiation on skin microbiota in patients with atopic dermatitis and healthy controls [Internet]. Vol. 67, International Journal of Circumpolar Health. 2008. p. 254-60. Available from: http://dx.doi.org/10.3402/ijch.v67i2-3.18282

49. Malanos D, Stern RS. Psoralen plus ultraviolet A does not increase the risk of cataracts: a 25year prospective study. J Am Acad Dermatol [Internet]. 2007 Aug;57(2):231-7. Available from: http://dx.doi.org/10.1016/j.jaad.2007.04.027

50. Rodríguez-Granados MT, Carrascosa JM, Gárate T, Gómez-Díez S, Guimaraens-Juantorena D. [Consensus document on bath-PUVA therapy. The Spanish Photobiology Group of the Spanish Academy of Dermatology and Venereology]. Actas Dermosifiliogr [Internet]. 2007 Apr;98(3):164-70. Available from: http://dx.doi.org/10.1016/sooo1-7310(07)70040-6

51. Nicolaidou E, Antoniou C, Stratigos AJ, Stefanaki C, Katsambas AD. Efficacy, predictors of response, and long-term follow-up in patients with vitiligo treated with narrowband UVB phototherapy. J Am Acad Dermatol [Internet]. 2007 Feb;56(2):274-8. Available from: http://dx.doi.org/10.1016/j.jaad.2006.09.004

52. Brazzelli V, Antoninetti M, Palazzini S, Barbagallo T, De Silvestri A, Borroni G. Critical evaluation of the variants influencing the clinical response of vitiligo: study of 60 cases treated with ultraviolet B narrow-band phototherapy [Internet]. Vol. 21, Journal of the European Academy of Dermatology and Venereology. 2007. p. 1369-74. Available from: http://dx.doi.org/10.1111/j.1468-3083.2007.02278.x

53. Jain VK, Bansal A, Aggarwal K, Jain K. Enhanced response of childhood psoriasis to narrow-band UV-B phototherapy with preirradiation use of mineral oil. Pediatr Dermatol [Internet]. 2008 Sep;25(5):559-64. Available from: http://dx.doi.org/10.1111/j.1525-1470.2008.00729.x

54. Imafuku $\mathrm{K}$, Hata $\mathrm{H}$, Yanagi $\mathrm{T}$, Kitamura $\mathrm{S}$, Inamura-Takashima Y, Nishimura M, Kitamura S, Moriwaki S, Shimizu H. Multiple skin cancers in patients with mycosis fungoides after long-term ultraviolet phototherapy. Clin Exp Dermatol [Internet]. 2017 Jul;42(5):523-6. Available from: http://dx.doi.org/10.1111/ced.13121

55. Lee E, Koo J, Berger T. UVB phototherapy and skin cancer risk: a review of the literature. Int J Dermatol [Internet]. 2005 May;44(5):355-60. Available from: http://dx.doi.org/10.1111/j.1365-4632.2004.02186.x

56. Kreuter A, Gambichler T, Avermaete A, Jansen T, Hoffmann M, Hoffmann K, Altmeyer P, VonKobyletzki G, Bacharach-Buhles M. Combined Treatment with Calcipotriol Ointment and Low-Dose Ultraviolet A1 Phototherapy in Childhood Morphea [Internet]. Vol. 18, Pediatric Dermatology. 2001. p. 241-5. Available from: http://dx.doi.org/10.1046/j.1525-1470.2001. 018003241.x

57. Fai D, Cassano N, Vena GA. Narrow-band UVB phototherapy combined with tacrolimus ointment in vitiligo: a review of 110 patients [Internet]. Vol. 21, Journal of the European Academy of Dermatology and Venereology. 2007. p. 916-20. Available from: http://dx.doi.org/10.1111/j.1468-3083.2006.02101.x 